\title{
APLICACIÓN DE LODOS DE PLANTA DE TRATAMIENTO DE CELULOSA: EFECTO EN ALGUNAS PROPIEDADES FÍSICAS Y QUÍMICAS DE SUELOS VOLCÁNICOS
}

\author{
Claudia Aravena R. ${ }^{1}$, Cristian Valentin C. ${ }^{1}$, M. Cristina Diez J. ${ }^{2}$, \\ M de la Luz MoraG. ' y Felipe Gallardo A. ${ }^{1}$ \\ ${ }^{1}$ Departameto de Ciencias Químicas, Universidad de La Frontera. Correo electrónico: \\ fgallar@ufro.cl \\ ${ }^{2}$ Departamento de Ingeniería Química Universidad de La Frontera. Casilla 54-D. Temuco. \\ Chile.
}

\section{Application of sludge from cellulose treatment plant: Effect in some physical and chemical properties of volcanic soils}

Key words: degraded soils, cellulose sludge, chemical and physical soil properties.

\begin{abstract}
The sludge from cellulose treatment plant is rich in organic matter (MO) and can be used to improve the chemical and physical properties of the soil. The aim of this study was to evaluate the effect of the application of sludge from cellulose wastewater treatment plant on the physical and chemical properties of volcanic soils (series Gorbea and Collipulli). There was added sludge $\left(0,10,20,30\right.$ and $\left.50 \mathrm{Mg} \mathrm{ha}^{-1}\right)$ to the soils and were incubated for 75 days at $22 \pm 2^{\circ} \mathrm{C}$. At 15 and 75 days the content of organic matter (MO), available nitrogen $(\mathrm{N}), \mathrm{pH}$, extractable $\mathrm{Al}$, field capacity, bulk density and aggregate stability was determinated. The application of sludge improved significantly $(\mathrm{p} \leq 0.05)$ the levels of nitrogen and organic matter in both soils, as well as the $\mathrm{pH}$, with the consistent decrease of Al level. On the other hand, the different doses of sludge did not improve significantly the physical properties of the soils, with the exception of the stability of the aggregates in the soil series Gorbea. The minor effect in the physical properties could happen due to the fact that the period of incubation (75 days) was not sufficient to provoke significant changes in the physical properties of the soils.
\end{abstract}

Palabras claves: Suelos degradados, celulosa, lodo, química de suelos, física de suelos 


\section{RESUMEN}

Los lodos de planta de tratamiento de celulosa son ricos en materia orgánica (MO) y pueden ser utilizados para mejorar las propiedades químicas y físicas del suelo. El objetivo de este estudio fue evaluar el efecto de la aplicación de lodo de planta de tratamiento de aguas residuales de la industria de celulosa sobre las propiedades físicas y químicas de suelos volcánicos (Serie Gorbea y Collipulli). Se adicionó lodo (0, 10, 20, 30 y $\left.50 \mathrm{Mg} \mathrm{ha}^{-1}\right)$ a los suelos y se incubó por 75 días a $22 \pm 2^{\circ} \mathrm{C}$. A los 15 y 75 días se determinó el contenido de materia orgánica (MO), nitrógeno disponible $(\mathrm{N}), \mathrm{pH}, \mathrm{Al}$ extractable, capacidad de campo, densidad aparente y estabilidad de los agregados. La aplicación de lodo incrementó significativamente $(\mathrm{p} \leq 0,05)$ los niveles de nitrógeno y de materia orgánica en ambos suelos, así como el pH, con la consecuente disminución del nivel de Al. Por otro lado, las distintas dosis de lodo no mejoraron significativamente las propiedades físicas de los suelos, a excepción de la estabilidad de los agregados en el suelo serie Gorbea. El menor efecto en las propiedades físicas pudo ocurrir debido a que el periodo de incubación (75 días) no fue suficiente para provocar cambios significativos en las propiedades físicas de los suelos.

\section{INTRODUCCIÓN}

La industria chilena de celulosa basada en el proceso Kraft, se ha desarrollado aceleradamente durante las útimas décadas. Este proceso genera grandes cantidades de aguas residuales, las cuales al ser tratadas por procesos biológicos, principalmente aerobios, generan lodos. Estos lodos están compuestos principalmente por compuestos orgánicos y su disposición constituye una preocupación económica y ambiental.

El lodo suele presentar contenidos de sólidos variable entre $0,25 \%$ y $12 \%$, dependiendo de las operaciones y procesos de tratamiento (Catricala et al., 1996). La estabilización de los lodos es el proceso por el cual disminuye la tasa de descomposición de la materia orgánica, dejando el material sin las condiciones necesarias de humedad y temperatura, evitando así una proliferación de microorganismos. Este proceso reduce el volumen del residuo, elimina la presencia de patógenos, reduce los compuestos tóxicos y olores (Winkler, 1986; Jokela et al., 1997). La aplicación de lodos a suelos agrícolas degradados puede ayudar a recuperar su productividad por aumento en el $\mathrm{pH}$, aumento en el contenido de materia orgánica y un mejoramiento de la disponibilidad de otros nutrientes tales como N, P y K. Sin embargo, la aplicación de lodos al suelo debe ser controlada, ya que altas dosis pueden provocar una disminución de la

densidad aparente y de las propiedades hidráulicas del suelo (Goyal et al., 1999) y una eventual acumulación de metales pesados.

En Chile, los estudios sobre la disposición de lodos para fines agrícolas y forestales son recientes, comparados con los realizados en Norte América y Europa. La disposición de lodos en suelos degradados o erosionados es una buena alternativa para Chile, considerando que en nuestro país $46 \%$ del suelo agrícola presenta erosión en tres niveles: seria, moderada y suave. Por otro lado, los suelos derivados de cenizas volcánicas ocupan en Chile un área aproximada a los 4 millones de hectáreas, de las cuales una superficie cercana al millón es arable. Estos suelos se caracterizan por poseer una alta capacidad de intercambio catiónico, alta capacidad de retención de fosfatos, bajo porcentaje de saturación de bases, altos contenidos de $\mathrm{Al}$ de intercambio y niveles de $\mathrm{pH}$ moderadamente ácidos a fuertemente ácidos (Gallardo et al., 1999; Mora, 1994).

La acidez de los suelos derivados de cenizas volcánicas limita el crecimiento de las plantas debido a una combinación de factores que incluyen, entre otros, elevados niveles de Al de intercambio (Baligar y Fageira, 1999; Gallardo et al., 1999). 
Además, es importante notar que el tratamiento de las aguas residuales en Chile aún no ha llegado a su producción máxima (Marambio y Ortega, 2003) por lo cual sólo se puede estimar la producción de los lodos, e inferir que representará un problema ambiental. El objetivo del presente trabajo fue evaluar el efecto de la aplicación de lodo proveniente del tratamiento de aguas residuales de la industria de celulosa, sobre las propiedades físicas y sobre el nivel de aluminio de intercambio, materia orgánica y nitrógeno en suelos agrícolas derivados de cenizas volcánicas.

\section{MATERIALES Y MÉTODOS Procedencia del lodo}

Se utilizó un lodo proveniente del tratamiento biológico (laguna aireada) del agua residual de una industria de celulosa kraft. El lodo es bombeado periódicamente desde la zona de sedimentación de la laguna a un landfill en donde permanecen alrededor de un año, en proceso de estabilización. Estudios previos realizados por Esparza (2004), demostraron que este lodo se encontraba estabilizado de acuerdo a ensayos de actividad biológica (ensayos de

Cuadro 1: Caracterización física y química del lodo: promedios y error estándar de la media.

Table 1: Physical and chemical characterization of the sludge: average and standard error of the mean.

\begin{tabular}{|c|c|c|}
\hline Parámetros & Unidades & Lodo \\
\hline Nitrógeno & $\left(\mathrm{mg} \mathrm{kg}^{-1}\right)$ & $586 \pm 6,6$ \\
\hline Fósforo & $\left(\mathrm{mg} \mathrm{kg}^{-1}\right)$ & $313 \pm 19,2$ \\
\hline $\mathrm{pH}$ & $\left(\mathrm{H}_{2} \mathrm{O}\right)$ & $6,97 \pm 0,2$ \\
\hline M. Orgánica & $(\%)$ & $76,1 \pm 1,0$ \\
\hline Potasio & $(\mathrm{cmol}+/ \mathrm{kg})$ & $3,62 \pm 0,31$ \\
\hline Sodio & $(\mathrm{cmol}+/ \mathrm{kg})$ & $41,6 \pm 1,7$ \\
\hline Calcio & $(\mathrm{cmol}+/ \mathrm{kg})$ & $28,0 \pm 1,6$ \\
\hline Magnesio & $(\mathrm{cmol}+/ \mathrm{kg})$ & $13,7 \pm 0,4$ \\
\hline Aluminio & $(\mathrm{cmol}+/ \mathrm{kg})$ & $0,03 \pm 0,00$ \\
\hline Suma Bases & $(\mathrm{cmol}+/ \mathrm{kg})$ & $86,8 \pm 4,0$ \\
\hline CICE & $(\mathrm{cmol}+/ \mathrm{kg})$ & $86,8 \pm 4,0$ \\
\hline Saturación de Al & (\%) & 0,035 \\
\hline Zinc & $\left(\mathrm{mg} \mathrm{kg}^{-1}\right)$ & $376,3 \pm 1,3$ \\
\hline Manganeso & $\left(\mathrm{mg} \mathrm{kg}^{-1}\right)$ & $111,1 \pm 2,0$ \\
\hline Cobre & $\left(\mathrm{mg} \mathrm{kg}^{-1}\right)$ & $5,04 \pm 0,05$ \\
\hline Hierro & $\left(\mathrm{mg} \mathrm{kg}^{-1}\right)$ & $18,5 \pm 0,1$ \\
\hline Conductividad & $\mathrm{dS} \mathrm{m}^{-1}$ & $16,7 \pm 0,8$ \\
\hline Humedad & $(\%)$ & $10,6 \pm 0,1$ \\
\hline
\end{tabular}


germinación y actividad respiratoria), indicando que puede usarse con seguridad en mezclas en suelos agrícolas. En el Cuadro 1 se presenta la caracterización fisicoquímica del lodo usado en este estudio. Este material presentó un contenido de materia orgánica de $76 \%$, como también altos contenidos de nutrientes disponibles como $\mathrm{N}$ y $\mathrm{P}$, equivalentes a 586 y $313 \mathrm{mg} \mathrm{kg}^{-1}$, respectivamente.

\section{Origen de los Suelos}

Los suelos seleccionados fueron suelos de las series Gorbea (Andisol) y Collipulli (Ultisol), muestreados en el horizonte superficial Ap a los $20 \mathrm{~cm}$ de profundidad. El suelo Gorbea presenta $\mathrm{pH}$ ácido $(4,8)$ y alto contenido de aluminio intercambiable $(0,9 \mathrm{cmol}+/ \mathrm{kg})$ mientras que el suelo Collipulli presenta un $\mathrm{pH}$ menos ácido $(5,6)$

Cuadro 2: Caracterización física y química de las series de suelo: promedios y error estándar de la media.

Table 2: Physical and chemical characterization of the soil series: average and standard error of the mean.

\begin{tabular}{|c|c|c|}
\hline Parámetros & Gorbea & Collipulli \\
\hline Humedad (\%) & $52,4 \pm 0,1$ & $9,2 \pm 0,1$ \\
\hline Capac. de Campo (mL $\left.100 \mathrm{~g}^{-1}\right)$ & $81,3 \pm 1,0$ & $57,2 \pm 0,1$ \\
\hline Densidad Aparente $\left(\mathrm{kg} \mathrm{m}^{3}\right)$ & $890,0 \pm 10$ & $1.060 \pm 50$ \\
\hline Estabilidad Agregados (\%) & $74,0 \pm 0,4$ & $51,9 \pm 0,8$ \\
\hline Porosidad (\%) & $57,0 \pm 0,3$ & $49,4 \pm 1,1$ \\
\hline Nitrógeno $\left(\mathrm{mg} \mathrm{kg}^{-1}\right)$ & $22,0 \pm 0,6$ & $18,2 \pm 1,6$ \\
\hline Fósforo $\left(\mathrm{mg} \mathrm{kg}^{-1}\right)$ & $11,9 \pm 0,3$ & $10,2 \pm 1,0$ \\
\hline $\mathrm{pH}\left(\mathrm{H}_{2} \mathrm{O}\right)$ & $4,8 \pm 0,02$ & $5,6 \pm 0,02$ \\
\hline Materia Orgánica (\%) & $15,1 \pm 0,13$ & $9,8 \pm 0,03$ \\
\hline Potasio $(\mathrm{cmol}+/ \mathrm{kg})$ & $0,2 \pm 0,01$ & $0,4 \pm 0,02$ \\
\hline Sodio $(\mathrm{cmol}+/ \mathrm{kg})$ & $0,07 \pm 0,003$ & $0,07 \pm 0,01$ \\
\hline Calcio $(\mathrm{cmol}+/ \mathrm{kg})$ & $0,5 \pm 0,02$ & $5,5 \pm 0,08$ \\
\hline Magnesio $(\mathrm{cmol}+/ \mathrm{kg})$ & $0,5 \pm 0,02$ & $1,3 \pm 0,09$ \\
\hline Aluminio $(\mathrm{cmol}+/ \mathrm{kg})$ & $0,9 \pm 0,01$ & $0,34 \pm 0,01$ \\
\hline Suma Bases $(\mathrm{cmol}+/ \mathrm{kg})$ & $1,2 \pm 0,03$ & $7,2 \pm 0,20$ \\
\hline $\mathrm{CICE}(\mathrm{cmol}+/ \mathrm{kg})$ & $2,1 \pm 0,04$ & $7,3 \pm 0,21$ \\
\hline Saturación de Al (\%) & 45,4 & 1,7 \\
\hline Zinc $\left(\mathrm{mg} \mathrm{kg}^{-1}\right)$ & $0,3 \pm 0,02$ & $0,2 \pm 0,01$ \\
\hline Manganeso (mg kg$\left.{ }^{-1}\right)$ & $5,0 \pm 0,16$ & $25,1 \pm 2,03$ \\
\hline Cobre $\left(\mathrm{mg} \mathrm{kg}^{-1}\right)$ & $1,1 \pm 0,05$ & $5,6 \pm 0,17$ \\
\hline Hierro $\left(\mathrm{mg} \mathrm{kg}^{-1}\right)$ & $34,7 \pm 1,46$ & $31,1 \pm 1,32$ \\
\hline
\end{tabular}


y menor contenido de materia orgánica (9,8\%) (Cuadro 2). Se obtuvieron dos porciones de suelo, una fue tamizada por 2 $\mathrm{mm}$ y secada al aire para su posterior caracterización y la otra porción se usó para los tratamientos de mezcla con lodos.

\section{Incubaciones}

Una vez realizadas las mezclas, las macetas fueron incubadas a $22 \pm 2{ }^{\circ} \mathrm{C}$ en cámara de crecimiento adaptadas para este propósito. Todos los suelos se mantuvieron a $50 \%$ de la capacidad de campo. Las evaluaciones se realizaron en dos oportunidades: al día 15 y 75 desde el comienzo de la incubación.

\section{Determinación de las propiedades físicas y químicas}

Se determinaron las siguientes propiedades físicas: estabilidad de los agregados (Kemper y Rosenau, 1986), capacidad de campo (Barajas et al., 1994), a través del método volumétrico, relacionando el porcentaje de humedad del suelo y la diferencia entre agua retenida y drenada después de 48 h. Además se determinó densidad aparente (Buckman, 1991), analizada por la relación pesovolumen. Los análisis químicos realizados fueron: materia orgánica, $\mathrm{N}$ disponible, $\mathrm{Al}$ extractable y pH según (Sadzawka et al., 2004).

\section{Diseño experimental y análisis estadístico}

El diseño experimental consistió en un arreglo factorial completamente al azar con cinco niveles de lodo: 0, 10, 20, 30 y 50 $\mathrm{Mg} \mathrm{ha}^{-1}$ en mezcla con $2 \mathrm{~kg}$ de suelo (base seca) y tres repeticiones. Los datos fueron analizados mediante una ANDEVA de una vía, considerando la prueba de comparación de medias de Tukey. También se utilizó la prueba $t$ student para muestras independientes con el objeto de comparar las medias en los tratamientos a 15 y 75 días. Ambas pruebas fueron ajustadas a un nivel de significación de $5 \%$. El programa estadístico utilizado corresponde a SPSS, V. 10.0.

\section{RESULTADOS Y DISCUSIÓN}

En el Cuadro 3 presenta el efecto de la aplicación del lodo sobre el contenido de materia orgánica (MO) en el suelo Gorbea y en el suelo Collipulli. Se observa que este parámetro incrementó significativamente especialmente en el suelo Collipulli por efecto de las dosis aplicadas, no observándose diferencia significativa $(p<0,05)$ en el suelo serie Gorbea en dosis igual o mayor a de $30 \mathrm{Mg} \mathrm{ha}^{-1}$. La aplicación de lodo equivalente a $50 \mathrm{Mg} \mathrm{ha}^{-1}$ correspondió a un $54 \%$ del total de MO del suelo Collipulli, por lo cual se observó un mayor efecto comparado con el suelo serie Gorbea en que la aplicación de $50 \mathrm{Mg} \mathrm{ha}^{-1}$ correspondió solo a un $17 \%$ del total de MO del suelo. Los incrementos de MO frente a la aplicación de lodo concuerdan con los resultados obtenidos por Diez et al., 1996; O'Brien et al., 2002; Gallardo et al., 2007 y Zhang et al., 2004. La materia orgánica junto a otros parámetros químicos como fertilidad del suelo, $\mathrm{pH}$, suma de bases, puede determinar la disponibilidad de los nutrientes presentes en el suelo y en consecuencia el rendimiento potencial de los cultivos (Honorato, 2000). 
Cuadro 3: Contenido de materia orgánica (\%) de los suelos Gorbea y Collipulli con 0, 10, 20, 30 y $50 \mathrm{Mg} \mathrm{ha}^{-1}$ de lodo de celulosa, después de 15 y 75 días de incubación.

Table 3: Organic matter content (\%) of Gorbea and Collipulli soils with 0, 10, 20, 30 and $50 \mathrm{Mg} \mathrm{ha}^{-1}$ of cellulose sludge, after 15 and 75 days of incubation.

\begin{tabular}{cccccc}
\hline $\begin{array}{c}\text { Tiempo de } \\
\text { incubación } \\
\text { (días) }\end{array}$ & 0 & 10 & 20 & 30 & 50 \\
\cline { 2 - 6 } & $13,07 \mathrm{bD}^{1}$ & $14,27 \mathrm{aC}$ & $15,06 \mathrm{aB}$ & $15,79 \mathrm{aA}$ & $15,98 \mathrm{aA}$ \\
15 & $13,95 \mathrm{aC}$ & $1,47 \mathrm{aB}$ & $15,68 \mathrm{aA}$ & $15,91 \mathrm{aA}$ & $16,05 \mathrm{aA}$ \\
75 & & & Suelo de lodo $\left(\mathrm{Mg} \mathrm{ha}^{-1}\right)$ \\
& & & & \\
& $10,39 \mathrm{aD}$ & $12,65 \mathrm{aC}$ & $14,67 \mathrm{aB}$ & $18,19 \mathrm{aA}$ & $19,26 \mathrm{aA}$ \\
15 & $10,77 \mathrm{aD}$ & $12,48 \mathrm{aC}$ & $14,49 \mathrm{aB}$ & $15,08 \mathrm{bB}$ & $16,81 \mathrm{bA}$ \\
\hline
\end{tabular}

'Para cada suelo por separado, los valores seguidos de distinta letra mayúscula en una misma fila y letra minúscula en una misma columna, presentan diferencias significativas $(\mathrm{p} \leq 0,05)$.

El contenido de nitrógeno disponible incrementó significativamente con la aplicación de lodo en ambos suelos estudiados, siendo mayor el efecto a medida que se incrementó la dosis de lodo aplicada (Cuadro 4). Se observó además diferencia significativa $(\mathrm{p}<0,05)$ entre los valores obtenidos a los 15 y a los 75 días de incubación. El efecto de la adición de lodo fue mayor en el suelo serie Gorbea, obteniéndose un contenido de $\mathrm{N}$ de 207,83 $\mathrm{mg} \mathrm{kg}^{-1}$ al día 75 para el tratamiento de 50 $\mathrm{Mg} \mathrm{ha}^{-1}$. Sin embargo, en el mismo periodo de incubación y en la misma dosis de lodo, el valor obtenido para el suelo serie Collipulli fue de $103,83 \mathrm{mg} \mathrm{kg}^{-1}$. Las variaciones entre ambos suelos se pueden deber a variación en la relación $\mathrm{C} / \mathrm{N}$ durante el período de incubación y a la diferente actividad biológica que presentan ambos suelos (Gallardo et al., 2006). Por otro lado, estudios realizados por Zhang et al., 2004 han determinado que después de dos meses de incubación, existe una disminución significativa de la lignina y de la disponibilidad de $\mathrm{C}$ y $\mathrm{N}$. 
Cuadro 4: Contenido de nitrógeno $\left(\mathrm{mg} \mathrm{kg}^{-1}\right)$ del suelo Gorbea y Collipulli con dosis de $0,10,20,30$ y $50 \mathrm{Mg} \mathrm{ha}^{-1}$ de lodo de celulosa, después de 15 y 75 días de incubación. Table 4: Nitrogen content $\left(\mathrm{mg} \mathrm{kg}^{-1}\right)$ of Gorbea and Collipulli soils with application of 0 , $10,20,30$ and $50 \mathrm{Mg} \mathrm{ha}^{-1}$ of cellulose sludge, after 15 and 75 days of incubation.

\begin{tabular}{cccccc}
\hline $\begin{array}{c}\text { Tiempo de } \\
\text { incubación } \\
\text { (días) }\end{array}$ & 0 & 10 & 20 & 30 & 50 \\
\cline { 2 - 6 } & & \multicolumn{5}{c}{ Suelo Gorbea } \\
15 & $24,61 \mathrm{bE}^{1}$ & $49,36 \mathrm{bD}$ & $56,1 \mathrm{bC}$ & $74,56 \mathrm{bB}$ & $100,77 \mathrm{bA}$ \\
75 & $28,77 \mathrm{aE}$ & $89,37 \mathrm{aD}$ & $103,32 \mathrm{aC}$ & $153,7 \mathrm{aB}$ & $207,83 \mathrm{aA}$ \\
& & & & & \\
& & & 5 & Suelo Collipulli & \\
75 & $18,04 \mathrm{aE}$ & $36,89 \mathrm{bD}$ & $64,00 \mathrm{aC}$ & $71,49 \mathrm{bB}$ & $82,05 \mathrm{bA}$ \\
75 & $19,06 \mathrm{aE}$ & $46,85 \mathrm{aD}$ & $57,53 \mathrm{aC}$ & $89,19 \mathrm{aB}$ & $103,83 \mathrm{aA}$ \\
\hline
\end{tabular}

Para cada suelo por separado, los valores seguidos de distinta letra mayúscula en una misma fila y letra minúscula en una misma columna, presentan diferencias significativas $(\mathrm{p} \leq 0,05)$.

El pH en los suelos Gorbea y Collipulli incrementó durante la incubación por efecto de la aplicación de lodo (Cuadro 5). En el suelo Gorbea el pH incrementa de 4,37 a 5,03 después de 15 días de incubación al aplicar lodo de $50 \mathrm{Mg} \mathrm{ha}^{-1}$ mientras que se obtiene un valor de $\mathrm{pH}$ de 4,76 al cabo de 75 días de incubación con la misma dosis aplicada. Similar situación se observa el en suelo Collipulli, en donde los valores de $\mathrm{pH}$ corresponden a 5,37 y 5,35 después de 15 y 75 días de incubación con la dosis de 50 $\mathrm{Mg} \mathrm{ha}^{-1}$, respectivamente. Se observa una disminución de los valores de $\mathrm{pH}$ en el periodo de incubación de 75 días, lo que concuerda con resultados obtenidos por Tang y Yu (1999), quienes señalan que el pH aumenta inmediatamente después de agregar el residuo, disminuyendo a través del tiempo. El aumento en los valores de pH está asociado al aumento en las bases de intercambio debido al aporte de $\mathrm{Na}^{+}, \mathrm{K}^{+}$, $\mathrm{Mg}^{+2}$ y Ca $^{+2}$ que se encuentran en cantidades apreciables en el lodo utilizado (Cuadro 1). Los cationes desplazan $\mathrm{H}^{+}$de los sitios de intercambio en el suelo, aumentando por lo tanto la saturación de bases. 
Cuadro 5: Valores de pH del suelo Gorbea y Collipulli con dosis de 0, 10, 20, 30 y 50 $\mathrm{Mg}$ ha $^{-1}$ de lodo de celulosa, después de 15 y 75 días de incubación.

Table 5: $\mathrm{pH}$ values of of Gorbea and Collipulli soils with application of 0, 10, 20, 30 and $50 \mathrm{Mg} \mathrm{ha}^{-1}$ of cellulose sludge, after 15 and 75 days of incubation.

\begin{tabular}{cccccc}
\hline $\begin{array}{c}\text { Tiempo de } \\
\text { incubación } \\
\text { (días) }\end{array}$ & 0 & 10 & 20 & 30 & 50 \\
\cline { 2 - 6 } & 4,37 & 4,47 & 4,67 & 4,79 & 5,03 \\
15 & 4,46 & 4,54 & 4,67 & 4,73 & 4,76 \\
75 & & & \multicolumn{5}{c}{ Suelo Gorbea } \\
& 5,06 & 5,17 & 5,21 & 5,30 & 5,37 \\
15 & 4,9 & 5,15 & 5,18 & 5,24 & 5,35 \\
75 & & \multicolumn{5}{c}{ Suelo Collipulli } \\
\hline
\end{tabular}

El incremento en el pH en suelos volcánicos reviste gran importancia ya que está relacionado con el nivel de $\mathrm{Al}$ intercambiable $\left(\mathrm{Al}^{+3}\right)$ en los suelos, el cual es tóxico para las plantas (Rengel, 2004). En suelos ácidos, el Al es liberado por el complejo de intercambio, debido a la disminución de bases y el aumento de iones hidrógeno. La aplicación de lodo proveniente del tratamiento de las aguas de la industria de celulosa a los suelos Gorbea y Collipulli aumentó los valores de $\mathrm{pH}$ con la consecuente disminución del Al de intercambio, tal como se muestra en la Figura 1 (a y b). Se observa que el nivel deAl disminuyó de 1,02 (cmol+/kg) a 0,23 $(\mathrm{cmol}+/ \mathrm{kg})$ a los 15 días de incubación en el Suelo Gorbea con $50 \mathrm{Mg} \mathrm{ha}^{-1}$. Este suelo presenta un mayor nivel de acidificación que el suelo Collipulli. Sin embargo, la adición de lodo también disminuyó el nivel de $\mathrm{Al}$ de intercambio, disminuyendo de 0,35 a 0,07 con la dosis de $50 \mathrm{Mg} \mathrm{ha}^{-1}$ después de 15 días de incubación. Resultados similares han sido obtenidos por Carpenter y Fernández (2000) y Zhang et al., (2004) al aplicar lodos de la industria de celulosa, obteniendo aumentos en los valores de $\mathrm{pH}$ y disminución en el \% de saturación de Al.

Los valores de capacidad de campo para el suelo Collipulli sin adición de lodo, son notoriamente inferiores a los obtenidos para la serie Gorbea (Cuadro 6). Sin embargo, existe un incremento significativo de este parámetro al incorporar dosis superiores a $20 \mathrm{Mg} \mathrm{ha}^{-1}$ para el suelo Collipulli. Este aumento se debe particularmente a que la aplicación de lodo como aporte en materia orgánica produce un incremento en la macroporosidad, con formación de agregados de mayor tamaño (ver más adelante), lo que se traduce en una mayor 

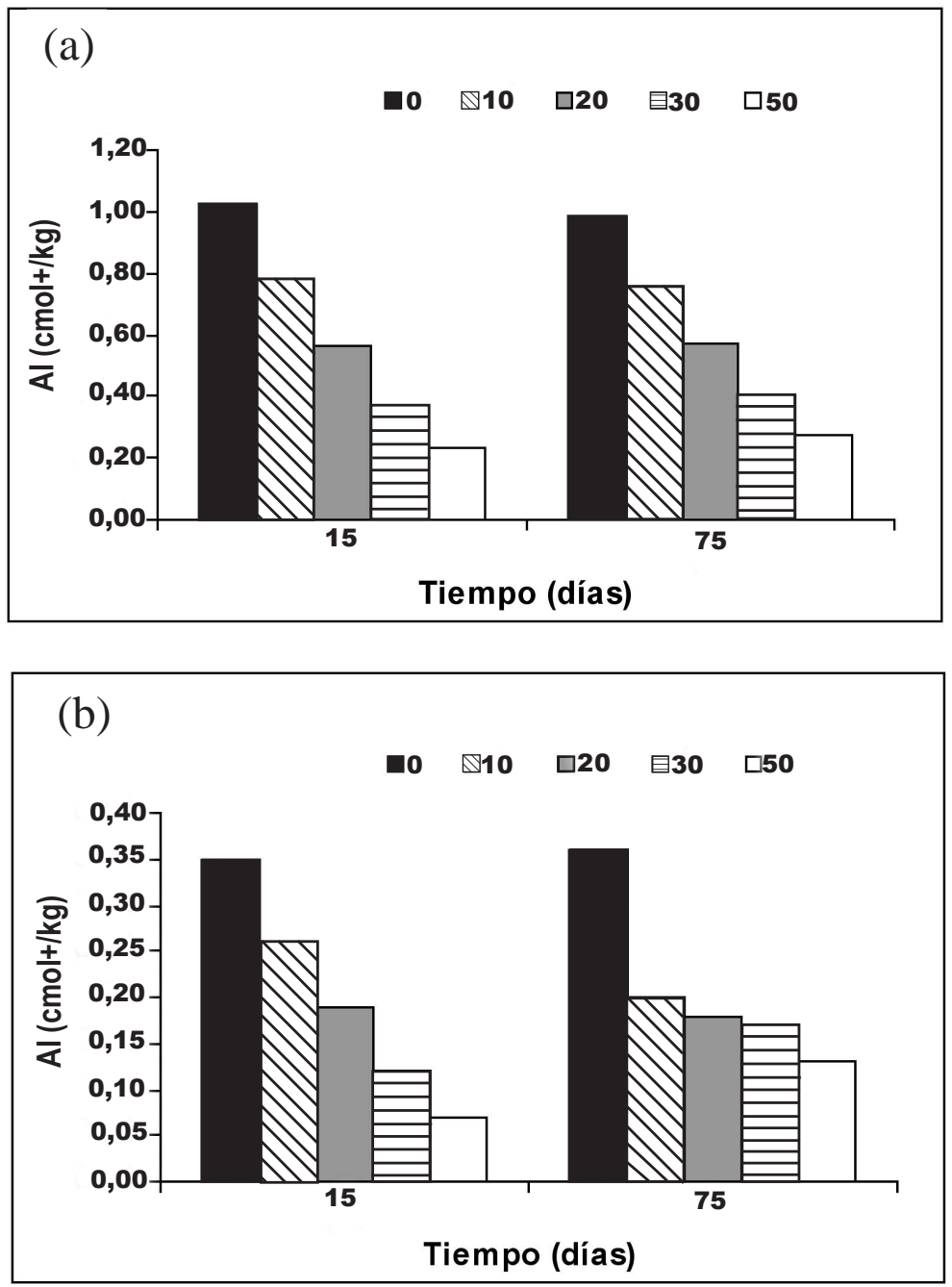

Figura 1: Efecto de la aplicación de lodo de celulosa (0, 10, 20, 30 and $50 \mathrm{Mg}^{-1}$ ) sobre el nivel de aluminio (cmol+/kg) en los suelos Gorbea (a) y Collipulli (b), después de 15 y 75 días de incubación.

Figure 1: Effect of the cellulose sludge application (0, 10, 20, 30 and $\left.50 \mathrm{Mg} \mathrm{ha}^{-1}\right)$ on aluminium content (cmol+/kg) in Gorbea (a) and Collipulli soils (b), after 15 and 75 days of incubation.

capacidad de retención de agua (Arias, 2003). Estudios similares realizados por Rivero et al. (2004), indican que el tiempo de incubación de dos meses no juega un papel fundamental, sin embargo, tiempos superiores permiten observar diferencias más marcadas. 


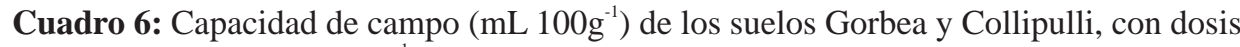
de $0,10,20,30$ y $50 \mathrm{Mg} \mathrm{ha}^{-1}$ de lodo de celulosa, después de 15 y 75 días de incubación.

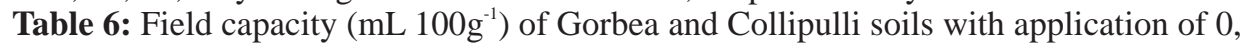
$10,20,30$ and $50 \mathrm{Mg} \mathrm{ha}^{-1}$ of cellulose sludge, after 15 and 75 days of incubation.

\begin{tabular}{cccccc}
\hline $\begin{array}{c}\text { Tiempo de } \\
\text { incubación } \\
\text { (días) }\end{array}$ & 0 & 10 & 20 & 30 & 50 \\
\cline { 2 - 6 } & & \multicolumn{5}{c}{ Suelo Gorbea } \\
15 & $82,48 \mathrm{bA}^{1}$ & $82,78 \mathrm{bA}$ & $84,74 \mathrm{aA}$ & $84,76 \mathrm{bA}$ & $85,33 \mathrm{aA}$ \\
75 & $83,17 \mathrm{aB}$ & $85,9 \mathrm{aA}$ & $86,08 \mathrm{aA}$ & $86,16 \mathrm{aA}$ & $86,24 \mathrm{aA}$ \\
& & & & \\
& & & Suelo Collipulli & \\
15 & $49,71 \mathrm{aAB}$ & $46,21 \mathrm{aB}$ & $49,68 \mathrm{aAB}$ & $52,53 \mathrm{bA}$ & $55,17 \mathrm{aA}$ \\
75 & $49,91 \mathrm{aB}$ & $49,06 \mathrm{aB}$ & $50,38 \mathrm{aB}$ & $55,02 \mathrm{aA}$ & $56,20 \mathrm{aA}$ \\
\hline
\end{tabular}

Para cada suelo por separado, los valores seguidos de distinta letra mayúscula en una misma fila y letra minúscula en una misma columna, presentan diferencias significativas $(\mathrm{p} \leq 0,05)$.

La estabilidad de los agregados no varió significativamente $(\mathrm{p}<0,05)$ en el suelo Collipulli, con el incremento en la dosis de lodo en ambos periodos evaluados, excepto entre el tratamiento control y la dosis de 50 $\mathrm{Mg} \mathrm{ha}^{-1}$ a los 75 días de incubación (Cuadro 7). Por otro lado, la estabilidad de los agregados incrementó en el suelo Gorbea a medida que se incorporó lodo como aporte en materia orgánica. Se observó diferencia significativa en el valor de la estabilidad de los agregados al aumentar la dosis de lodo en ambos periodos de evaluación, obteniendose un incremento superior a un $25 \%$ al aplicar $50 \mathrm{Mg} \mathrm{ha}^{-1}$ de lodo (Cuadro 7). Sin embargo, no se observó diferencia significativa entre ambos periodos de evaluación al aplicar dosis igual o menor a $20 \mathrm{Mg} \mathrm{ha}^{-1}$. Estudios similares fueron obtenidos por Betancourt et al. (1999), donde la materia orgánica presentó correlaciones positivas para la estabilidad de los agregados, presentando un mejoramiento en las condiciones físicas de suelos degradados, principalmente en su estabilidad estructural. Es así como las aplicaciones de lodo generan un aumento en la estabilidad de los agregados. Aportes de hasta un $5 \%$ de lodos urbanos generan aumentos de hasta un $78 \%$ de la estabilidad de agregados respecto a la condición inicial de un suelo con 7,5 g $\mathrm{kg}^{-1}$ de materia orgánica (Guerrero et al., 2001), lo que asegura un potencial de protección del suelo ante la erosión (Roldán et al., 1996). La mayor estabilidad se traduciría en un aumento del diámetro medio de los agregados, favoreciendo el movimiento del agua y aire (Trelo-Ges y Chuasavathi, 2002). Además, la redistribución del sistema poroso tendría un efecto positivo, mejorando la capacidad de retención de agua (Muñoz et al., 1999). 
Cuadro 7: Estabilidad de los agregados al agua (\%) de los suelos Gorbea y Collipulli, con dosis de $0,10,20,30$ y $50 \mathrm{Mg} \mathrm{ha}^{-1}$ de lodo de celulosa, después de 15 y 75 días de incubación.

Table 7: Water aggregate stability (\%) of Gorbea and Collipulli soils with application of $0,10,20,30$ and $50 \mathrm{Mg} \mathrm{ha}^{-1}$ of cellulose sludge, after 15 and 75 days of incubation.

\begin{tabular}{|c|c|c|c|c|c|}
\hline \multirow{2}{*}{$\begin{array}{c}\text { Tiempo de } \\
\text { incubación } \\
\text { (días) }\end{array}$} & \multicolumn{5}{|c|}{ Dosis de lodo $\left(\mathrm{Mg} \mathrm{ha}^{-1}\right)$} \\
\hline & 0 & 10 & 20 & 30 & 50 \\
\hline & \multicolumn{5}{|c|}{ Suelo Gorbea } \\
\hline 15 & $58,42 \mathrm{aD}^{1}$ & $67,5 \mathrm{aC}$ & $68,18 \mathrm{aC}$ & $72,52 \mathrm{bB}$ & $82,45 \mathrm{bA}$ \\
\hline \multirow[t]{2}{*}{75} & $62,06 \mathrm{aD}$ & $69,16 \mathrm{aC}$ & $70,16 \mathrm{aC}$ & $78,16 \mathrm{aB}$ & $84,89 \mathrm{aA}$ \\
\hline & \multicolumn{5}{|c|}{ Suelo Collipulli } \\
\hline 15 & $61,81 \mathrm{aA}$ & $62,11 \mathrm{aA}$ & $62,06 \mathrm{aA}$ & $62,5 \mathrm{bA}$ & $65,05 \mathrm{aA}$ \\
\hline 75 & $61,98 \mathrm{aB}$ & $63,03 \mathrm{aAB}$ & $63,21 \mathrm{aAB}$ & $64,55 \mathrm{aAB}$ & $65,69 \mathrm{aA}$ \\
\hline
\end{tabular}

Para cada suelo por separado, los valores seguidos de distinta letra mayúscula en una misma fila y letra minúscula en una misma columna, presentan diferencias significativas $(\mathrm{p} \leq 0,05)$.

En general no se observaron variaciones significativas en función de la tasa de aplicación del lodo para la densidad aparente, a excepción del suelo Collipulli con dosis superiores a $20 \mathrm{Mg} \mathrm{ha}^{-1}$ después de 75 días de incubación (Cuadro 8). La densidad aparente disminuyó en dosis altas de lodo pero este efecto no se expresó en el suelo Gorbea. Estos resultados contrastan con los obtenidos por Seguel et al., (2003). Según estudios realizados por Goyal et al., (1999), la presencia de materia orgánica afecta considerablemente la densidad aparente en el largo plazo, sin embargo, se debe tener en cuenta que las aplicaciones de enmiendas orgánicas aumentan la agregación y con ello, la porosidad fina. $\mathrm{Si}$ esta nueva porosidad no muestra un nivel adecuado de continuidad y funcionalidad, al realizar los análisis de laboratorio, el agua que queda retenida tenderá a provocar una sobreestimación de los valores iniciales de porosidad gruesa. Es fundamental determinar cuales son los efectos, tanto cualitativos como cuantitativos de las aplicaciones de lodos sobre las propiedades físicas y mecánicas de los suelos. 
Cuadro 8: Densidad aparente $\left(\mathrm{kg} \mathrm{m}^{-3}\right)$ de los suelos Gorbea y Collipulli con dosis de 0 , $10,20,30$ y $50 \mathrm{Mg} \mathrm{ha}^{-1}$ de lodo de celulosa, después de 15 y 75 días de incubación. Table 8: Bulk density $\left(\mathrm{kg} \mathrm{m}^{-3}\right)$ of Gorbea and Collipulli soils with application of 0,10 , 20,30 and $50 \mathrm{Mg} \mathrm{ha}^{-1} \mathrm{~s}$ of cellulose ludge, after 15 and 75 days of incubation.

\begin{tabular}{cccccc}
\hline $\begin{array}{c}\text { Tiempo de } \\
\text { incubación } \\
\text { (días) }\end{array}$ & 0 & 10 & 20 & 30 & 50 \\
\cline { 2 - 6 } & & \multicolumn{5}{c}{ Suelo Gorbea } \\
15 & $0,89 \mathrm{aA}^{1}$ & $0,88 \mathrm{aA}$ & $0,88 \mathrm{aA}$ & $0,88 \mathrm{aA}$ & $0,87 \mathrm{aA}$ \\
75 & $0,88 \mathrm{aA}$ & $0,86 \mathrm{aA}$ & $0,86 \mathrm{aA}$ & $0,83 \mathrm{aA}$ & $0,83 \mathrm{aA}$ \\
& & \multicolumn{5}{c}{ Suelo Collipulli } \\
& & & & \\
15 & $1,2 \mathrm{aA}$ & $1,17 \mathrm{aA}$ & $1,14 \mathrm{aA}$ & $1,05 \mathrm{aA}$ & $1,04 \mathrm{aA}$ \\
75 & $1,2 \mathrm{aA}$ & $1,13 \mathrm{aA}$ & $1,09 \mathrm{aAB}$ & $0,97 \mathrm{aB}$ & $0,98 \mathrm{aB}$ \\
\hline
\end{tabular}

'Para cada suelo por separado, los valores seguidos de distinta letra mayúscula en una misma fila y letra minúscula en una misma columna, presentan diferencias significativas $(\mathrm{p} \leq 0,05)$.

\section{CONCLUSIONES}

El lodo como enmienda es una alternativa para mejorar las propiedades físicas y químicas en suelos agrícolas. Esto permite predecir un mejor desarrollo del cultivo, teniendo en cuenta el método y tiempo de aplicación, propiedades del lodo y del suelo. La aplicación de lodo mejoró significativamente los niveles de nitrógeno y materia orgánica en ambos suelos, así como, incrementó los valores de $\mathrm{pH}$, con la consecuente disminución del contenido de $\mathrm{Al}$ de intercambio. Sin embargo, las distintas dosis de lodo no mejoraron significativamente las propiedades físicas de los suelos, a excepción de la estabilidad de los agregados en el suelo Gorbea, lo cual puede deberse al corto periodo de tiempo en el cual se realizó el ensayo (75 días).

\section{AGRADECIMIENTOS}

Este Trabajo fue financiado por el Fondo Nacional de Investigación Científica y Tecnológica FONDECYT N¹040854. Agradecemos muy especialmente al Dr. Francisco Matus, académico del Departamento de Ciencias Químicas de la Universidad de La Frontera por los numerosos comentarios tanto de redacción como de contenido en la versión original de este manuscrito. 


\section{BIBLIOGRAFÍA}

ARIAS M. 2003. "Utilización de residuos sólidos industriales provenientes del proceso de fabricación de celulosa kraft en la producción vegetal". Trabajo para optar al título de Ingeniero Ambiental. Universidad de La Frontera, Temuco.

BARAJAS G., HART L. AND BROOKER, M. 1994. Laboratory manual of the soil microbial biomass group.

BALIGAR, V.C. AND FAGEIRA, N.K. 1999. Plant nutrient efficiency: towards the second paradigm. Sociedade Brasileira de Ciências do Solo.183-205.

BETANCOURT P., GONZÁLEZ J., FIGUEROA B. Y GONZÁLEZ F. 1999. Materia orgánica y caracterización de suelos en proceso de recuperación con coberturas vegetativas en zonas templadas de México. México.

BUCKMAN A. Naturaleza y propiedades de los suelos. 1991. Editorial Limusa. D. F., México, 42-54, 415

CARPENTER, A. AND FERNANDEZ, I. 2000. Pulp. Sludge as a component in manufactured topsoil. J. Environ. Qual. 29:387-397.

CATRICALA C.E., BOWDEN W.B., SMITH C.T. AND MCDOWEFI W.H. 1996. Chemical characteristics of leachate from pulp and papermill residuals used to reclaim a sandy soil. Water, Air and Soil Pollution 89:167187.

DIEZ M.C., CONCHA M.I. Y GALLARDO F. 1996. Acid soil supplementation with sewage sludge for cereal growth. IV International Symposium on PlantSoil Interactions at low pH. Belo Horizonte, Brasil.
ESPARZA J. 2004. “Uso de lodo biológico proveniente del tratamiento de aguas de la industria de la celulosa como mejorador de suelos con alto grado de degradación". Tesis presentada para optar al grado académico de magíster en ciencias de recursos naturales. Universidad de La Frontera, Temuco. Chile.

GALLARDO, F., BRAVO, C., ARAVENA, C. Y DIEZ, M.C. 2006. Aplicación de diferentes niveles de lodo secundario proveniente de la industria de celulosa a suelos degradados y su efecto sobre propiedades biológicas. XXX Congreso Interamericano de Ingeniería Sanitaria y Ambiental (AIDIS). Punta del Este, Uruguay. GALLARDO, F., MORA, M.L AND. DIEZ, M.C. 2007. Kraft mill sludge to improve vegetal production in Chilean Andisol. Water Science and Technology (in press).

GUERRERO C., MATAIX-SOLERA J., NAVARRO-PEDREÑO J., GARCÍA-ORENES F. AND GÓMEZ I. 2001. Different patterns of aggregate stability in burned and restored soils. Arid Land Research and Management 15: 163-171.

GOYAL S., CHANDER K., MUNDRA M.C. AND KAPOOR K.K. 1999. Influence of inorganic fertilizers and organic amendments on soil organic matter and soil microbial properties under tropical conditions. Biology and Fertility of Soils 29:196-200.

HONORATO R. 2000. Manual de edafología. Cuarta edición. Ediciones Universidad Católica de Chile. Santiago-Chile. 195p. 
JOKELA J., RINTALA J., OIKARI A., REINIKAINEN O., MUTKA K., AND NYRONEN T. 1997. Aerobic composting and anaerobic digestion of pulp and paper mill sludge. Water Science Technology 36(11):181-188. KEMPER W.D. AND ROSENAU R.C. 1986. Agreggate stability and distribution. In: Methods of soil analysis. Part I. Physical and mineralogical methodos; Klute A. (Ed). American Society of Agronomy and Soil Science Society of America: Madison, W1.:425-442.

MARAMBIO C. AND ORTEGA R. 2003. Uso potencial de lodos derivados del tratamiento de aguas servidas en la producción de cultivos en Chile. Revista Agronomía y Forestal UC. 20: 20-23.

MORA, M.L. 1993. Nivel de fertilidad de los suelos de la IX región y su relación con la acidez. Frontera Agrícola 1(1): 5-12.

MUÑOZ F.; POLO M. Y GIRADLES J. 1999. Modificación física de algunas propiedades físicas en un suelo del Valle del Guadalquivir enmendado con lodos de depuradora, en Estudios de la zona no saturada del suelo, pp115-121. Muñoz R., Ritter A. y Tascón, C. (Ed). ICIA. Tenerife.

O' BRIEN T.A., HERBERT S.J. AND BARKER A.V. 2002. Growth of corn in varying mixtures of paper mill sludge and soil. Communications in Soil Science and Plant Analysis 33(3\&4): 635-646.

RENGEL, Z. 2004. Aluminium cycling in the soil-plant-animal-human continuum. Biometals 17(6):669689.

RIVERO C., LOBO D. Y LÓPEZ A. 2004. Efectos de la incorporación de residuos orgánicos sobre algunas propiedades físicas de un Alfisol degradado. Universidad Central de Venezuela, Facultad de Agronomía, Instituto de Edafología. Apdo. 4579. Maracay, Ed. Aragua. Particular. Venezuela.
ROLDÁN A., ALBADALEJO J. AND THORNES J.B. 1996. Aggregate stability changes in a semiarid soil after treatment with different organic amendments. Arid Soil Research Rehabilitation 10:139-148.

SADZAWKA A., CARRASCO M., GREZ R. Y MORA M. 2004. Métodos de análisis recomendados para los suelos chilenos. Comisión de normalización y acreditación. Sociedad Chilena de la Ciencia del Suelo.

SEGUEL O., GARCÍA DE CORTÁZAR V. Y CASANOVA M. 2003. Variación en el tiempo de las propiedades físicas de un suelo con adición de enmiendas orgánicas. Agricultura Técnica. 63(3):1-15.

TANG, C AND YU, Q. 1999. Impact of chemical composition of legume residues and initial soil $\mathrm{pH}$ on $\mathrm{pH}$ change of a soil after residue incorporation. Plant and Soil (215):29-38

TRELO-GES V. AND CHUASAVATHI T. 2002. Effect of municipal waste and grass cultivation on physical properties of a sandy soil of northeast Thailand. p. 924. In: Abstracts of 17th World Congress of Soil Science, 14-17 August 2002, Bangkok, Thailand.

WINKLER M. 1986. Tratamiento Biológico de Aguas de Desecho. Editorial Limusa S.A. de C.V., México D.F., México. 188 p.

ZHANG S., WANG S., SHAN X. AND MU H. 2004. Influences of lignin from paper mill sludge on soil properties and metal accumulation in wheat. Biology and Fertility of Soils 40:237242. 